\title{
Exergy analysis of R717 high-efficiency OTEC power cycle for the efficiency and pressure drop in main components
}

\author{
Jung-In Yoon*, Chang-Hyo Son* ${ }^{* \dagger}$, Dong-II Yang**, Hyeon-Uk Kim*, Hyeon-Ju \\ $\mathrm{Kim}^{\star \star *}$ and Ho-Saeng Lee ${ }^{\star \star *}$
}

(received 17 September 2013, revised 30 September 2013, accepted 30 September 2013)

\begin{abstract}
In this paper, an analysis on exergy efficiency of high-efficiency R717 OTEC power system for the efficiency and pressure drop in main components were investigated theoretically in order to optimize the design for the operating parameters of this system. The operating parameters considered in this study include turbine and pump efficiency, and pressure drop in a condenser and evaporator, respectively. As the turbine efficiency of R717 OTEC power system increases, the exergy efficiency of this system increases. But pressure drop in the evaporator of R717 OTEC power system increases, the exergy efficiency of this system decreases, respectively. And, in case of exergy efficiency of this OTEC system, the turbine efficiency and pressure drop in a condenser on R717 OTEC power system is the largest and the lowest among operation parameters, respectively.
\end{abstract}

Key Words : R717, High-Efficiency, Ocean Thermal Energy Conversion(OTEC) Power Cycle, Exergy Analysis, Pressure drop

\section{Introduction}

For environmental issues such as global warming and ozone depletion, interest has been focused on the development of technologies that take advantage of renewable energy, such as ocean energy. Especially, in spite of the low efficiency

\footnotetext{
$* \dagger$ Chang-Hyo Son(corresponding author) : Department of Refrigeration and Air Conditioning Engineering, Pukyong National University.

E-mail: sonch@pknu.ac.kr, Tel: 051-621-6183

*Jung-In Yoon : Department of Refrigeration and Air Conditioning Engineering, Pukyong National University.

**Dong-Il Yang and Hyeon-Uk Kim : Graduate School of Air Conditioning Engineering, Pukyong National University.

***Hyeon-Ju Kim and Ho-Saeng Lee : Korea Advanced Institute of Science and Technology.
}

of ocean thermal energy conversion (following OTEC), the OTEC has some advantages such as the ability to be used as a clean energy source, obtained directly from the ocean surface water and deep water. In a situation, the research related to the OTEC is being done in various worldwide.

The OTEC uses the temperature difference between cooler deep and warmer shallow or surface ocean waters to run a heat engine and produce useful work, usually in the form of electricity. Our country has progressed diversified research with an interest in the development of OTEC power generation systems to industryuniversity joint research from $20100^{1-2)}$

Many researchers ${ }^{3-6)}$ have been much effort for the development of high-efficiency OTEC power 
system. Uehara et al. ${ }^{5)}$ such as the Kalina et al. ${ }^{\text {) }}$ used the ammonia-water mixture as a working fluid, applied multi-stage turbine, reheater, separators and other devices to OTEC power cycle. This cycle represents a similar efficiency of OTEC power cycle with reheater. But, the cycle is required precise optimal control in order to highly efficient operation.

In this study, as shown in Figure 1, high-efficiency OTEC power cycle with cooler, reheater, expansion valve and gas-liquid separator is developed. The cycle reduces the dissipation heat of condenser by adopting the gas-liquid separator and cooler, and the absorption heat of evaporator by using the reheater, and increases the power output and efficiency by applying two-stage turbine.

Therefore, the high-efficiency OTEC power cycle to grasp its exergy characteristics using HYSYS $^{7)}$ software. And, the exergy effects on each variable of the OTEC power cycle are analyzed. Accordingly, it is intended to provide a basis design data of highly efficient OTEC power cycle proposed in this study.

\section{Performance analysis}

\subsection{Schematic diagram}

Fig. 1 shows the schematic of the highefficiency R717 OTEC power cycle proposed in this study. As shown in Fig. 1, this OTEC cycle consists of two turbines, one pump, four heat exchangers, one gas-liquid separator and one expansion valve. R717(ammonia) as a working fluid is used. Furthermore, the high-temperature surface seawater and the low-temperature deep seawater are used for the evaporation and condensation of the working fluid that circulates inside the evaporator and the condenser, respectively.
To examine the flow of the working fluid that circulates inside this OTEC power cycle, the working fluid with a high temperature and pressure that evaporated from the evaporator enters the high-stage turbine and produces stored work around it by expanding to the medium pressure before cooling while passing through the regenerator and the cooler.

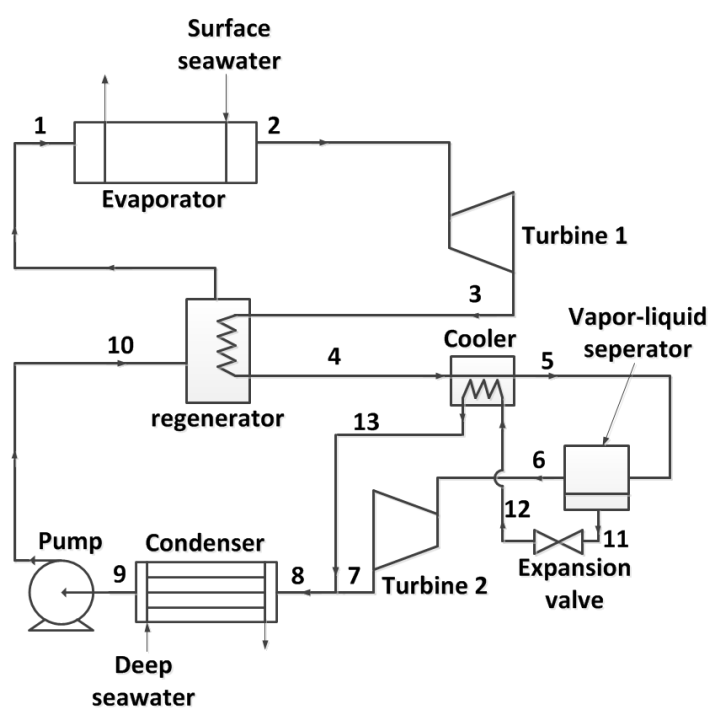

Fig. 1 Schematic of high-efficiency R717 OTEC power cycle proposed in this study ${ }^{2)}$

As stated, the OTEC power cycle that is proposed in this study reduces the discharged heat in the condenser by adopting a cooler and a gas-liquid separator, decreases the absorbed heat using a regenerator and increases the output work and the system efficiency through a two-stage turbine with high and low stages.

\subsection{Performance analysis}

The balance equations of the irreversibility and exergy destruction factor (EDF) for each component of the OTEC power cycle are as follows.

Evaporator : 
Exergy analysis of R717 high-efficiency OTEC power cycle for the efficiency and pressure drop in main components

Table 1: Analysis range of high-efficiency R717 OTEC power cycle proposed in this study

\begin{tabular}{|c|c|}
\hline Refrigerant & $\mathrm{R} 717\left(\mathrm{NH}_{3}\right)$ \\
\hline Evaporation pressure drop, $\Delta \mathrm{P}_{\mathrm{e}},[\mathrm{kPa}]$ & $5^{*}, 15,25,35,45$ \\
\hline Condensation pressure drop, $\Delta \mathrm{P}_{\mathrm{c}},[\mathrm{kPa}]$ & $5^{*}, 15,25,35$ \\
\hline Regenerator pressure drop, $\Delta \mathrm{P}_{\mathrm{HX} 1},[\mathrm{kPa}]$ & $1350,(\mathrm{~W}=20 \mathrm{~kW})$ \\
\hline Mass flow rate, $\mathrm{m},[\mathrm{kg} / \mathrm{h}]$ & $0.55,0.65,0.75,0.85^{*}, 0.95$ \\
\hline Turbine efficiency, $\eta_{\mathrm{t}},[/]$ & $0.55,0.65,0.75,0.85^{*}, 0.95$ \\
\hline Pump efficiency, $\eta_{\mathrm{p}},[/]$ & $22^{*}$ \\
\hline Surface seawater outlet temperature, $\mathrm{T}_{\mathrm{ss}, \text { out }},\left[{ }^{\circ} \mathrm{C}\right]$ & $66^{*}$ \\
\hline Deep seawater outlet temperature, $\mathrm{T}_{\mathrm{ds}, \text { out }},\left[{ }^{\circ} \mathrm{C}\right]$ & 134,000 \\
\hline Surface seawater mass flow rate, $\mathrm{m}_{\mathrm{ss}},[\mathrm{kg} / \mathrm{h}]$ & $\mathrm{x}=0$ \\
\hline Deep seawater mass flow rate, $\mathrm{m}_{\mathrm{ds}},[\mathrm{kg} / \mathrm{h}]$ & $630^{*}$ \\
\hline Condenser outlet vapor quality, $\mathrm{x}_{\mathrm{c}, \text { out }},[/]$ & $535^{*}$ \\
\hline High turbine outlet pressure, $\mathrm{P}_{\mathrm{HT}, \text { out }},[\mathrm{kPa}]$ & $915^{*}$ \\
\hline Condenser inlet pressure, $\mathrm{P}_{\mathrm{c}, \text { in }},[\mathrm{kPa}]$ & $0.95^{*}$ \\
\hline Evaporator outlet pressure, $\mathrm{P}_{\mathrm{e}, \mathrm{in}},[\mathrm{kPa}]$ & \\
\hline Cooler outlet vapor quality, $\mathrm{x}_{\mathrm{co}, \mathrm{out}},[/]$ & $* \mathrm{Reference}$ value \\
\hline
\end{tabular}

$$
\begin{aligned}
& \Delta \mathrm{ex}_{\text {loss }, \mathrm{e}}=\mathrm{m}\left(\mathrm{ex}_{1}-\mathrm{ex}_{2}\right)+\mathrm{Q}_{\mathrm{e}}\left(1-\mathrm{T}_{\mathrm{o}} / \mathrm{T}_{\mathrm{H}}\right) \\
& \mathrm{EDF}_{\mathrm{e}}=\Delta \mathrm{ex}_{\text {loss }, \mathrm{e}} / \mathrm{W}
\end{aligned}
$$

Condenser :

$$
\begin{aligned}
& \Delta \mathrm{ex}_{\text {loss }, \mathrm{c}}=\mathrm{m}\left(\mathrm{ex}_{1}-\mathrm{ex}_{2}\right)-\mathrm{Q}_{\mathrm{c}}\left(1-\mathrm{T}_{\mathrm{o}} / \mathrm{T}_{\mathrm{L}}\right) \\
& \mathrm{EDF}_{\mathrm{c}}=\Delta \mathrm{ex}_{\text {loss }, \mathrm{c}} / \mathrm{W}
\end{aligned}
$$

Heat exchanger :

$$
\begin{aligned}
& \Delta \mathrm{ex}_{\text {loss }, \mathrm{HX}}=\sum_{\mathrm{i}=1}^{\mathrm{n}} \mathrm{m}_{\mathrm{i}}\left(\mathrm{ex}_{\mathrm{i}, \mathrm{in}}-\mathrm{ex}_{\mathrm{i}, \text { out }}\right) \\
& \mathrm{EDF}_{\mathrm{HX}}=\Delta \mathrm{ex}_{\text {loss }, \mathrm{HX}} / \mathrm{W}
\end{aligned}
$$

Turbine :

$\triangle \mathrm{ex}_{\text {loss }, \mathrm{t}}=\mathrm{m}\left(\mathrm{ex}_{1}-\mathrm{ex}_{2}\right)-\mathrm{W}_{\mathrm{t}}$$$
\mathrm{EDF}_{\mathrm{t}}=\Delta \mathrm{ex}_{\text {loss }, \mathrm{t}} / \mathrm{W}
$$

Pump :

$$
\Delta \mathrm{ex}_{\text {loss }, \mathrm{p}}=\mathrm{m}\left(\mathrm{ex}_{1}-\mathrm{ex}_{2}\right)+\mathrm{W}_{\mathrm{p}}
$$

$$
\mathrm{EDF}_{\mathrm{p}}=\Delta \mathrm{ex}_{\text {loss }, \mathrm{p}} / \mathrm{W}
$$

Tank :

$\Delta \mathrm{ex}_{\text {loss }, \mathrm{ta}}=\mathrm{m}_{1} \mathrm{ex}_{1}-\mathrm{m}_{2} \mathrm{ex}_{2}-\mathrm{m}_{3} \mathrm{ex}_{3}$

$\mathrm{EDF}_{\mathrm{ta}}=\Delta \mathrm{ex}_{\text {loss, ta }} / \mathrm{W}$

mixer :

$\Delta \mathrm{ex}_{\text {loss }, \mathrm{mx}}=\mathrm{m}_{1} \mathrm{ex}_{1}+\mathrm{m}_{2} \mathrm{ex}_{2}-\mathrm{m}_{3} \mathrm{ex}_{3}$

$\mathrm{EDF}_{\mathrm{mx}}=\Delta \mathrm{ex}_{\text {loss }, \mathrm{mx}} / \mathrm{W}$

Expansion valve :

$$
\begin{aligned}
& \Delta \mathrm{ex}_{\text {loss,exp }}=\mathrm{m}\left(\mathrm{ex}_{1}-\mathrm{ex}_{2}\right) \\
& \mathrm{EDF}_{\text {exp }}=\Delta \mathrm{ex}_{\text {loss,exp }} / \mathrm{W}
\end{aligned}
$$

For the same conditions, the second law efficiency of the OTEC power cycle, $\eta_{\Pi}$, is a function of a ratio of real efficiency, $\eta_{\text {real }}$, to maximum reversible efficiency, $\eta_{\text {th,rev }}$. 


$$
\eta_{\text {II }}=\frac{\eta_{\text {real }}}{\eta_{\text {th, rev }}}=\frac{\eta_{\text {OTEC }}}{1-\mathrm{T}_{\mathrm{o}} / \mathrm{T}_{\mathrm{H}}}
$$

Where, $\mathrm{T}_{\mathrm{H}}$ is $25^{\circ} \mathrm{C}$ as a heat source temperature, $\mathrm{T}_{\mathrm{o}}$ is $30^{\circ} \mathrm{C}$ as an ambient temperature of OTEC power cycle using R717. Thus, the second law efficiency is a negative value, because of $T_{0} / T_{H}$ in Eq. (17) is higher than one. In this case, the second law efficiency can be expressed from an EDF exergy destruction factor) and defined as Eq. (18). ${ }^{8)}$

$$
\eta_{\text {II }}=\frac{1}{1+\mathrm{EDF}_{\text {total }}}
$$

Where, $\mathrm{EDF}_{\text {total }}$ is the sum of the EDFs for four main components in the subcritical OTEC power cycle.

Using the results of the calculation of the analysis conditions in Table 1. such factors as the turbine and pump efficiency, the pressure drop of the evaporator and condenser, which affect the exergy efficiency of the proposed R717 OTEC power cycle, were examined.

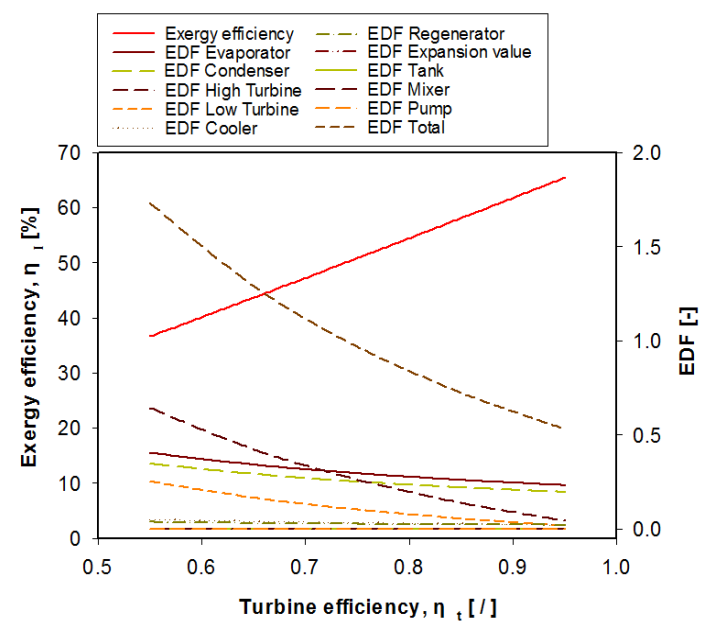

Fig. 2 Exergy efficiency and EDF of R717 OTEC power cycle with turbine efficiencies

\section{Results and discussion}

To find the medium pressure (the high-stage turbine outlet pressure) at which the efficiency of the proposed high-efficiency OTEC power cycle peaks, the changes in the evaporation heat capacity, the condensation heat capacity and the total work were examined while the outlet pressure of the high-stage turbine were examined. Therefore, the existence of a turbine outlet pressure that shows the highest efficiency was confirmed. That is, the high-efficiency OTEC power cycle has the highest efficiency of $4.25 \%$ at the high-stage turbine outlet pressure of $630 \mathrm{kPa}$. So, the exergy efficiency of the proposed OTEC power cycle was analyzed using the turbine outlet pressure of $630 \mathrm{kPa}$.

Fig. 2 shows the exergy efficiency $\left(\eta_{\Pi}\right)$ and exergy destruction factor(EDF) with respect to the variation of high- and low-stage turbine efficiency $\left(\eta_{\mathrm{t}}\right)$ of R717 OTEC power cycle under conditions. From Fig. 2, the exergy efficiency increases about $79 \%$ with the turbine efficiency due to the increasing net working power of high-and low-stage turbine. And, the EDFs of high- and low-stage turbine, condenser and evaporator decrease with the increasing turbine efficiency, respectively. This is the irreversibility of high- and low-stage turbine, condenser and evaporator. Therefore, the exergy efficiency of OTEC power cycle increases because of the decrease in their EDFs and $\mathrm{EDF}_{\text {total }}$.

Fig. 3 presents the exergy efficiency and EDF of R717 OTEC power cycle according to the change of pump efficiency $\left(\eta_{\mathrm{p}}\right)$ under the analysis conditions. In Fig. 3, the exergy efficiency of R717 OTEC power cycle increases with the pump efficiency. The reason is because of only $0.27 \mathrm{~kW}$ compared to the net turbine power of $20 \mathrm{~kW}$. From the above results, the $\mathrm{EDF}_{\mathrm{p}}$ of pump is 
smaller than that of other devices. So, the exergy efficiency of OTEC power cycle has no effect on the variation of pump efficiency. And, $\mathrm{EDF}_{\text {total }}$ does not change due to the constant EDF of other devices except for refrigerant pump.

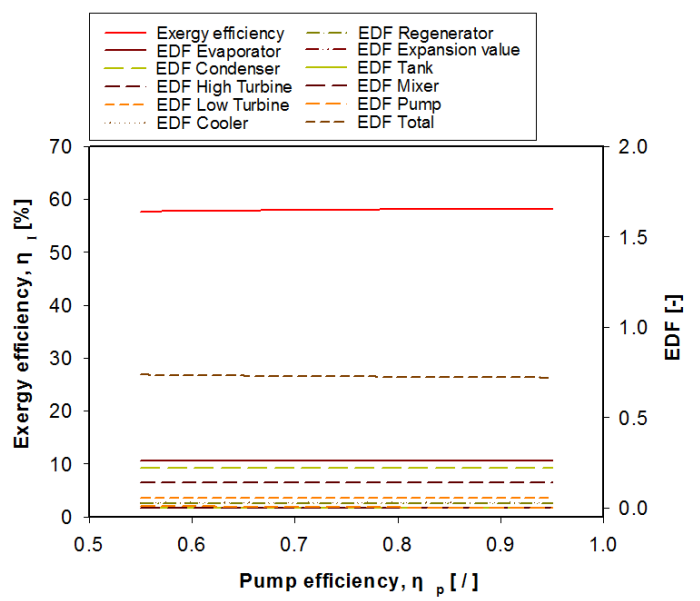

Fig. 3 Exergy efficiency and EDF of R717 OTEC power cycle with pump efficiencies

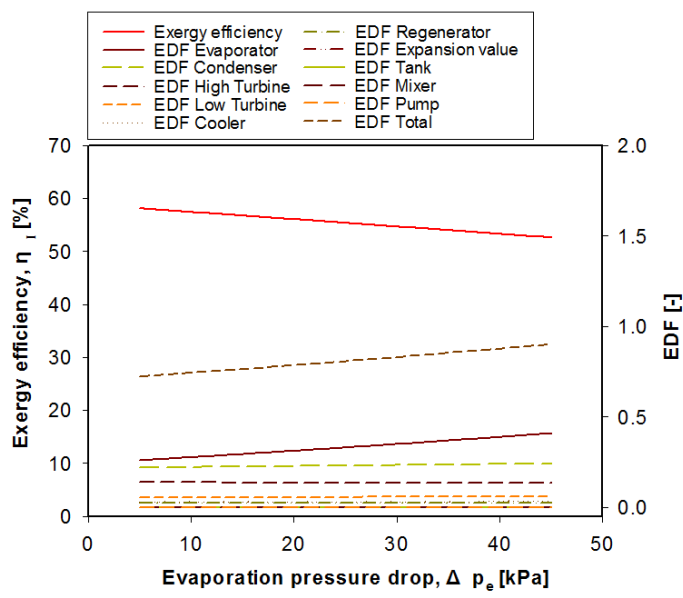

Fig. 4 Exergy efficiency and EDF of R717 OTEC power cycle with pressure drops in evaporator

Fig. 4 depicts the exergy efficiency and EDF of R717 OTEC power cycle according to the change of refrigerant pressure drop in the evaporator $\left(\Delta \mathrm{P}_{\mathrm{e}}\right)$ under the analysis conditions. As shown in Fig. 4, the exergy efficiency of OTEC power cycle decreases with the increase of pressure drop in the evaporator. This is because the inlet pressure of high-stage turbine decreases with the increasing pressure drop, resulting in decreasing the power of high-stage turbine. The exergy efficiency of OTEC power cycle decreases with the increasing pressure drop in the evaporator, due to the increase of evaporator EDF, as shown in Eq. (18).

Fig. 5 displays the exergy $\operatorname{efficiency}\left(\eta_{\mathrm{II}}\right)$ and EDF of R717 OTEC power cycle according to the variation of refrigerant pressure drop in the condenser $\left(\Delta \mathrm{P}_{c}\right)$ under the analysis conditions. From Fig. 5, the exergy efficiency has a constant with the pressure drop in the condenser. The outlet pressure of condenser(also inlet pressure of refrigerant pump) decreases as the pressure drop increases. From the above results, the EDF and power of pump increase, respectively. At the time, the EDF of pump has not an effect on the exergy efficiency of OTEC power cycle. And the total EDF has a constant value due to the constant EDF of other devices. Therefore, the exergy efficiency of OTEC power cycle has a constant value.

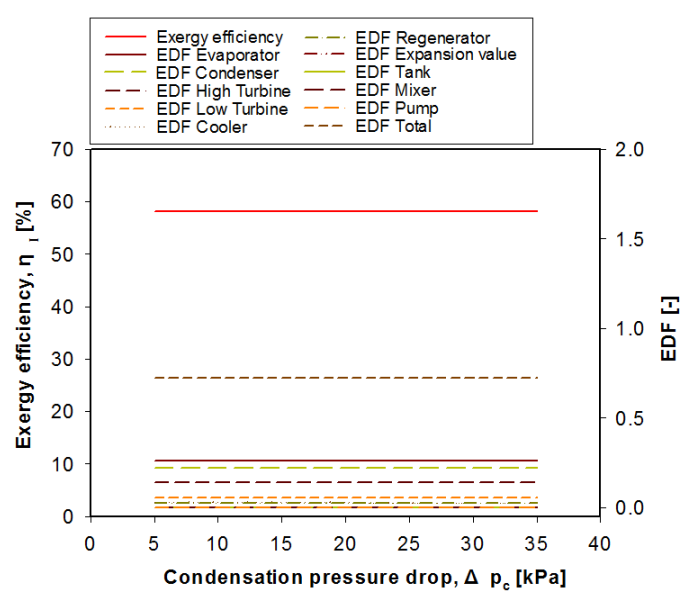

Fig. 5 Exergy efficiency and EDF of R717 OTEC power cycle with pressure drops in condenser 


\section{Conclusions}

So far, the exergy efficiency and EDF of the proposed R717 OTEC power cycle with respect to the variation of turbine efficiency $\left(\eta_{\mathrm{t}}\right)$, pump $\operatorname{efficiency}\left(\eta_{\mathrm{p}}\right)$ and pressure drop in the evaporator and condenser are investigated theoretically using HYSYS software under analysis conditions. The findings can be summarized as follows.

The exergy efficiency of R717 OTEC power cycle increases with turbine efficiency, but decrease with the pressure drop in the evaporator. Therefore, the exergy efficiency has a more effect on turbine efficiency compared to the pressure drop in the heat exchanger. From the above results, it is an important to develop the high-efficiency turbine in order to enhance the exergy efficiency of the proposed R717 OTEC power cycle. And, the exergy efficiency of the system should be identified by grasping the exergy loss of each component. The exergy loss of the R717 OTEC power cycle should be minimized to indicate its maximum exergy efficiency.

\section{Acknowledgement}

This work was financially supported by the National R\&D project of the "Development of Energy utilization technology with Deep Ocean Water" supported by the Korean Ministry of Land, Transport and Maritime Affairs.

\section{References}

1. H. S. Lee, H. J. Kim, D. H. Jung, et al., 2011, "A Study on the Improvement for Cycle Efficiency of Closed-type OTEC", Journal of the Korea Society of Marine Engineering, vol. 25 , no. 1 , pp. $80-84$.

2. J. H. Heo, C. H. son, J. I. Yoon, et al., 2013,
"Performance Characteristics of a R744 OTEC Power System Using High Temperature Heat Source", The Korean Society of Marine Engineering.

3. N. J. Kim, C. N. Kim, W. Chun, 2009, "Using the condenser effluent from a nuclear power plant for Ocean Thermal Energy Conversion (OTEC)", International Communications in Heat and Mass Transfer, vol. 36, pp. 1008-1013.

4. T. J. Rabas, C. B. Panchal, H. C. Stevens, 1990, "Integration and optimization of the gas removal system for hybrid-cycle OTEC power plants", J. Solar Energy Eng, vol. 112, pp. 19-28.

5. H. Uehara, A. Miyara, Y. Ikegami, T. Nakaoka, 1996, "Performance analysis of an OTEC plant and a desalination plant using an integrated hybrid cycle", J. Solar Energy Eng, vol. 118, pp. 115-122.

6. A. I. Kalina, 1984, "Combined cycle system with novel bottoming cycle", Journal of Engineering for Gas Turbines and Power, vol. 106, no. 4, pp. 737-742.

7. Aspen HYSYS_V8.0, Aspen Technology Inc.

8. Z. Shengjun, W. Huaixin, G. Tao, 2011, "Performance comparison and parametric optimization of subscritical organic rankine cycle(ORC) and transcritical power cycle system for low-temperature geothermal power generation", Applied energy, vol. 88, pp. 2740-2754. 